\title{
The SMAD-binding domain of SKI: a hotspot for de novo mutations causing Shprintzen-Goldberg syndrome
}

\author{
Dorien Schepers ${ }^{1,20}$, Alexander J Doyle ${ }^{2,3,20}$, Gretchen Oswald ${ }^{2}$, Elizabeth Sparks ${ }^{2}$, Loretha Myers ${ }^{2}$, Patrick J Willems ${ }^{4}$, \\ Sahar Mansour ${ }^{5}$, Michael A Simpson ${ }^{6}$, Helena Frysira ${ }^{7}$, Anneke Maat-Kievit ${ }^{8}$, Rick Van Minkelen ${ }^{8}$, \\ Jeanette M Hoogeboom ${ }^{8}$, Geert R Mortier ${ }^{1}$, Hannah Titheradge ${ }^{9}$, Louise Brueton ${ }^{9}$, Lois Starr ${ }^{10}$, Zornitza Stark ${ }^{11}$, \\ Charlotte Ockeloen ${ }^{12}$, Charles Marques Lourenco ${ }^{13}$, Ed Blair ${ }^{14}$, Emma Hobson ${ }^{15}$, Jane Hurst ${ }^{16}$, Isabelle Maystadt ${ }^{17}$, \\ Anne Destrée $^{17}$, Katta M Girisha ${ }^{18}$, Michelle Miller ${ }^{19}$, Harry C Dietz ${ }^{2,3}$, Bart Loeys ${ }^{1,20}$ and Lut Van Laer ${ }^{\star, 1,20}$
}

\begin{abstract}
Shprintzen-Goldberg syndrome (SGS) is a rare, systemic connective tissue disorder characterized by craniofacial, skeletal, and cardiovascular manifestations that show a significant overlap with the features observed in the Marfan (MFS) and Loeys-Dietz syndrome (LDS). A distinguishing observation in SGS patients is the presence of intellectual disability, although not all patients in this series present this finding. Recently, SGS was shown to be due to mutations in the SKI gene, encoding the oncoprotein SKI, a repressor of TGF $\beta$ activity. Here, we report eight recurrent and three novel SKI mutations in eleven SGS patients. All were heterozygous missense mutations located in the R-SMAD binding domain, except for one novel in-frame deletion affecting the DHD domain. Adding our new findings to the existing data clearly reveals a mutational hotspot, with $73 \%$ (24 out of 33 ) of the hitherto described unrelated patients having mutations in a stretch of five SKI residues (from p.(Ser31) to p.(Pro35)). This implicates that the initial molecular testing could be focused on mutation analysis of the first half of exon 1 of SKI. As the majority of the known mutations are located in the R-SMAD binding domain of SKI, our study further emphasizes the importance of TGF $\beta$ signaling in the pathogenesis of SGS.
\end{abstract}

European Journal of Human Genetics (2015) 23, 224-228; doi:10.1038/ejhg.2014.61; published online 16 April 2014

\section{INTRODUCTION}

Shprintzen-Goldberg syndrome (SGS; MIM 182212) is a rare, multisystemic connective tissue disorder characterized by craniosynostosis, severe skeletal muscle hypotonia, and intellectual disability. Common features observed in SGS patients include craniofacial (craniosynostosis, proptosis, dolichocephaly, hypertelorism, high arched palate, downslanting palpebral fissures, and retrognathia), skeletal (arachnodactyly, camptodactyly, scoliosis, pectus deformity, and joint hypermobility), cardiovascular (mitral valve prolaps and aortic dilatations), and neuromuscular (infantile hypotonia and intellectual disability) anomalies. As such, a considerable clinical overlap with Marfan syndrome (MFS; MIM 154700) and Loeys-Dietz syndrome (LDS; LDS1A: MIM 609192, LDS1B: MIM 610168, LDS2A: MIM 608967, LDS2B: MIM 610380, LDS3: MIM 613795, LDS4: MIM 190220) exists. ${ }^{1,2}$ During the last decade, it has become increasingly clear that dysregulated transforming growth factor beta (TGF $\beta$ ) signaling is having a major role in the pathogenesis of MFS, LDS, and related disorders involving thoracic aortic aneurysms. ${ }^{3-9}$ Because of the overlap with MFS and LDS and because several lines of evidence have confirmed a key role of TGF $\beta$ signaling in the pathogenesis of MFS and LDS, it was hypothesized that altered TGF $\beta$ signaling also underlies the SGS pathogenesis. Indeed, de novo, heterozygous mutations in SKI (Sloan-Kettering Institute), encoding a known repressor of TGF $\beta$ signaling, were identified in 10 SGS patients. ${ }^{10}$ The causal nature of SKI mutations was confirmed in two additional cohorts of SGS patients, in which two in-frame deletions and twelve missense mutations were identified. ${ }^{11,12}$ All SKI mutations clustered in two distinct $\mathrm{N}$-terminally located regions of the protein: the R-SMAD binding domain and the Dachshund-homology domain (DHD).

SKI is an avian sarcoma viral oncogene homolog that is located on chromosome 1 and consists of seven exons. Its gene product counts

${ }^{1}$ Center for Medical Genetics, Faculty of Medicine and Health Sciences, University of Antwerp and Antwerp University Hospital, Antwerp, Belgium; ${ }^{2}$ McKusick-Nathans Institute of Genetic Medicine, Johns Hopkins University School of Medicine, Baltimore, MD, USA; ${ }^{3}$ Howard Hughes Medical Institute, Baltimore, MD, USA; ${ }^{4}$ GENDIA, GENetic DIAgnostic Network, Antwerp, Belgium; ${ }^{5}$ SW Thames Regional Genetics Service, St George's, University of London, London, UK; ${ }^{6}$ Division of Genetics and Molecular Medicine, Department of Medical and Molecular Genetics, King's College London School of Medicine, London, UK; ${ }^{7}$ Department of Medical Genetics, National and Kapodistrian University of Athens Medical School, Athens, Greece; ${ }^{8}$ Department of Clinical Genetics, Erasmus MC, University Medical Center, Rotterdam, The Netherlands; ${ }^{9}$ Department of Clinical Genetics, Birmingham Women's Hospital, Birmingham, UK; ${ }^{10} \mathrm{Clinical}$ Genetics, Munroe-Meyer Institute for Genetics and Rehabilitation, Nebraska Medical Center, Omaha, NE, USA; ${ }^{11}$ Victorian Clinical Genetics Services, Murdoch Childrens Research Institute, Parkville, Victoria, Australia; ${ }^{12}$ Department of Human Genetics, Radboud University Nijmegen Medical Center, Nijmegen, The Netherlands; ${ }^{13}$ Department of Medical Genetics, School of Medicine of Ribeirao Preto, University of Sao Paulo, Sao Paulo, Brazil; ${ }^{14}$ Department of Clinical Genetics, Churchill Hospital, Oxford, UK; ${ }^{15}$ Department of Clinical Genetics, Chapel Allerton Hospital, Leeds, UK; ${ }^{16}$ Department of Clinical Genetics, Great Ormond Street Hospital, London, UK; ${ }^{17}$ Center for Human Genetics, Institute for Pathology and Genetics (IPG), Gosselies, Belgium; ${ }^{18}$ Division of Medical Genetics, Department of Pediatrics, Kasturba Medical College, Manipal University, Manipal, India; ${ }^{19}$ Department of Cardiology, All Childrens Hospital, St. Petersburg, FL, USA

*Correspondence: Professor L Van Laer, Center for Medical Genetics, University of Antwerp and Antwerp University Hospital, Prins Boudewijnlaan 43,2650 Antwerp, Belgium. Tel: +32 3275 9759; Fax: +32 3275 9722; E-mail: lut.vanlaer@uantwerpen.be

20These authors contributed equally to this work.

Received 16 October 2013; revised 24 February 2014; accepted 5 March 2014; published online 16 April 2014 
728 amino acids and functions as a repressor of TGF $\beta$ signaling by inhibiting R-SMAD (SMAD2-3) phosphorylation, hereby preventing assembly of the active heteromeric R-SMAD/SMAD4 complex and translocation to the nucleus. ${ }^{13-15}$ Additionally, the SKI protein can recruit transcriptional corepressors including SNW1, N-CoR, and mSIN3 and forms a complex with histone deacetylases. ${ }^{16,17}$ In vivo studies in Xenopus embryos, zebrafish, and mice have shown that SKI has a critical role in the development of neuronal and muscle cells or tissues. ${ }^{18-20}$ Homozygous Ski-targeted mice show a major reduction in skeletal muscle mass and defects in cranial neural tube closure, manifestations that resemble those observed in SGS patients. ${ }^{19}$ These experiments suggest a critical role for SKI in the development of muscle, craniofacial structures, and the central and peripheral nervous system, providing additional evidence for the pathogenic character of SKI mutations in SGS.

Here, we report on the identification of ten additional SKI mutations, three novel and seven previously reported, in eleven unpublished SGS patients. Except one, all mutations are located in the R-SMAD binding domain of SKI, confirming the importance of this domain. Moreover, our new data reveal a mutational hotspot, with $73 \%$ of the hitherto described patients having mutations affecting four SKI residues.

\section{MATERIALS AND METHODS}

Study participants

In total, 19 patients with clinically suspected SGS were recruited from the Howard Hughes Medical Institute (Baltimore, MD, USA), the Johns Hopkins University School of Medicine (Baltimore, MD, USA), the SW Thames Regional Genetics Service, St George's (London, UK), the King's College London School of Medicine (London, UK), the National and Kapodistrian University of Athens Medical School (Athens, Greece), the Birmingham Women's Hospital (Birmingham, UK), Gendia (Antwerp, Belgium), the Erasmus Medical Center (Rotterdam, The Netherlands), Nebraska Medical Center (Omaha, USA), Murdoch Childrens Research Institute (Parkville, Australia), Radboud University Nijmegen Medical Center (Nijmegen, The Netherlands), School of Medicine of Ribeirao Preto (Sao Paulo, Brazil), Churchill Hospital (Oxford, UK), Chapel Allerton Hospital (Leeds, UK), Institute for Pathology and Genetics (Gosselies, Belgium), Kasturba Medical College (Manipal, India), and All Childrens Hospital (Florida, USA). Informed consent was obtained from all patients or their families.

\section{Mutation analysis and Sanger sequencing}

DNA was extracted from blood using standard procedures. PCR was performed using standard conditions on a GeneAmp PCR System 2700 thermal cycler (Applied Biosystems, Foster City, CA, USA). PCR products were bidirectionally sequenced using the BigDye Terminator Cycle Sequencing kit (Applied Biosystems) and separated on an ABI 3130XL Genetic Analyzer in accordance with the manufacturer's instructions (Applied Biosystems). Primer sequences and conditions have been described previously. ${ }^{10}$ Sequences were analyzed using the CLC Sequence Viewer (CLC bio, Aarhus, Denmark). Sequence comparison and numbering are based on Ensembl transcript ENST00000378536 (Refseq NM_003036.3).
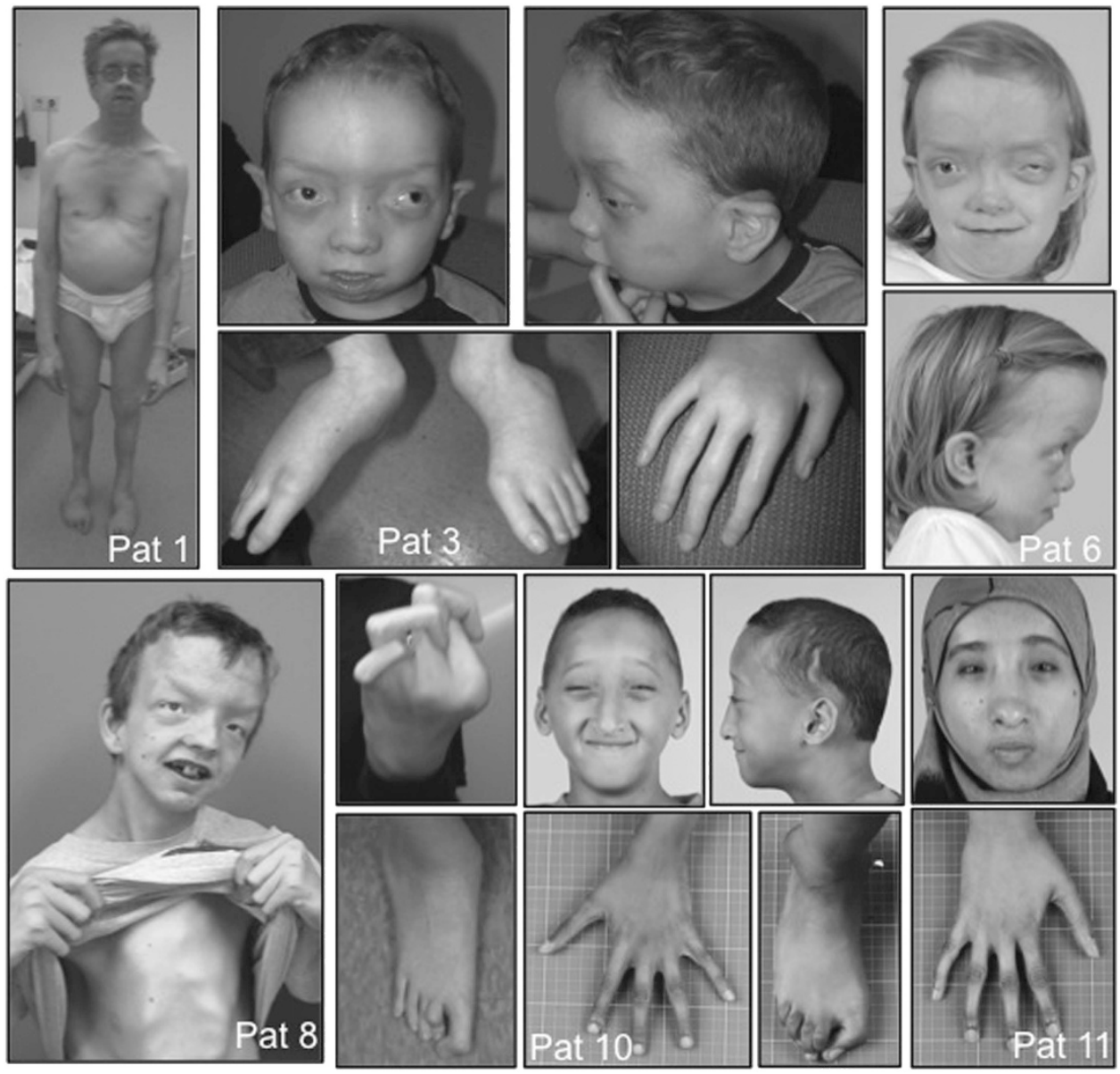

Figure 1 Clinical pictures of SGS patients. Note typical craniofacial features including craniosynostotic skull with proptosis, dolichocephaly with retrognathia, low set ears, and hypertelorism. Skeletal findings include arachnodactyly, scoliosis, and a combination of joint hyperlaxity and contractures with camptodactyly. 


\section{RESULTS}

Sanger sequencing of the complete coding region of SKI in nineteen patients with a clinical picture compatible with SGS revealed eight different, heterozygous missense mutations in addition to an in-frame deletion of $12 \mathrm{bp}$ (Figures 1 and 2) in eleven patients. The p.(Pro35Ser) mutation occurred in two unrelated patients (pat. 4 and 6) and the p.(Gly34Asp) was found in two siblings (pat. 10 and 11). We could prove the de novo occurrence of the mutations in five patients. For patient 8 only the mother, in whom the mutation was absent, could be tested. No DNA was available of the father of patient 8 , nor of the parents of the patients $1-3$. We did not find any evidence of somatic mosaicism in the blood of the parents of the siblings (pat. 10 and 11), thus germline mosaicism is the most likely explanation for the occurrence of two diseased children from healthy parents. Six different mutations have been described previously, ${ }^{10,11}$ while three were novel (p.(Ser28Thr), p.(Gly34Ala), and p.(Ser97_Arg100del)). Except the p.Ser97_Arg100 in-frame deletion, which was positioned in the DHD domain, all mutations clustered in the R-SMAD binding domain of SKI (Figure 2). Besides the de novo occurrence, we considered the following facts as additional proof of causality: (1) six out of nine different mutations had previously been described as disease causing; (2) the mutations were not present in the 1000 Genomes project, in dbSNP135, or in more than 10000 alleles of the National Heart, Lung and Blood Institute (NHLBI) Exome Variant Server; (3) all mutations affected highly conserved SKI residues; (4) MutationTaster, SIFT, and Polyphen categorized the eight missense mutations as disease causing, not tolerated and as possibly or probably damaging, respectively. All variants described in this paper are online available at the SKI locus-specific database on the HGVS website.

The clinical picture of the 11 patients having an SKI mutation is compatible with previously reported patients. Typical craniofacial features include craniosynostosis with proptosis, dolichocephaly with retrognathia, hypertelorism, low set ears, and the absence of ectopia lentis. Skeletal findings include arachnodactyly, scoliosis, and a combination of joint hyperlaxity and contractures with camptodactyly. Except one, all patients had invariable mild-to-moderate developmental delay and intellectual disability. As seen in previous studies some patients had mild aortic root dilatation. Table 1 and Figure 1 summarize the clinical picture of the 11 SGS patients. Most patients who were negative for SKI mutations had atypical clinical features (see Supplementary Table 1).

\section{DISCUSSION}

As shown in this and three previous reports, ${ }^{10-12}$ mutations in SKI cause SGS, a disorder that is usually classified among the syndromic forms of thoracic aortic aneurysms, more specifically among the MFS- and LDS-related disorders. The majority of the patients described within our first paper had dilatations of the aortic root, with z-scores of at least 2.0. ${ }^{10}$ Within the current series only two patients presented with aortic root dilatation, the hallmark of MFS and LDS. The difference between these two reports may be due to selection bias that may have occurred for the first screened set of SGS a

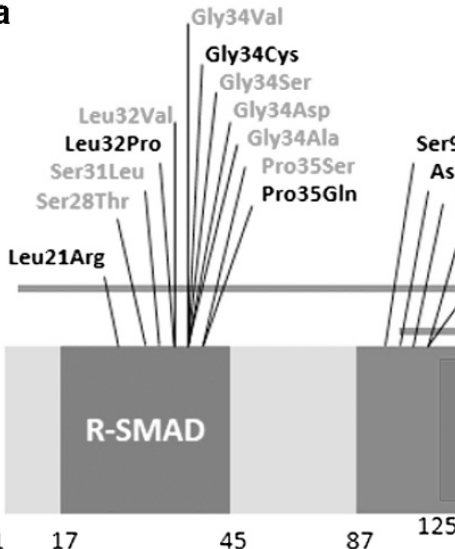

117

45 87
Ser94_Ser97del

Asp95_Ser97del

Ser97_Arg100del Gly116Glu Gly117Arg $\mathrm{N}-\mathrm{COR}$

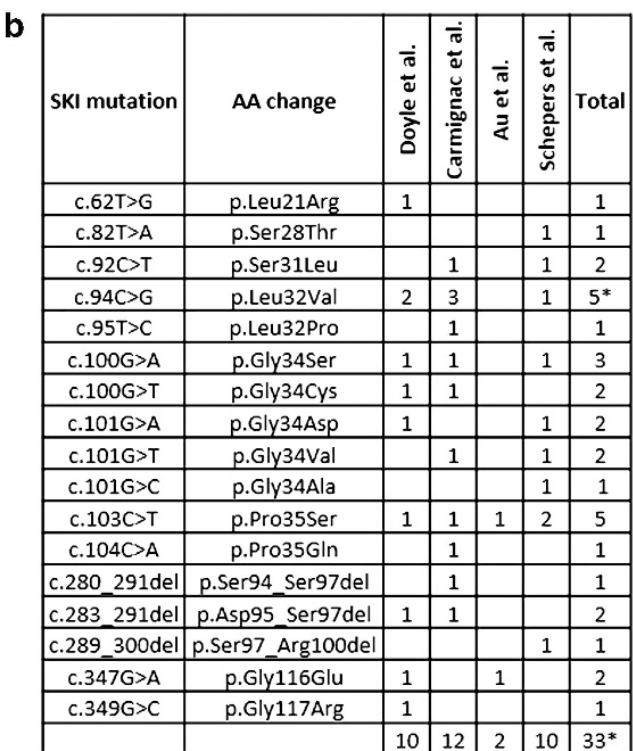

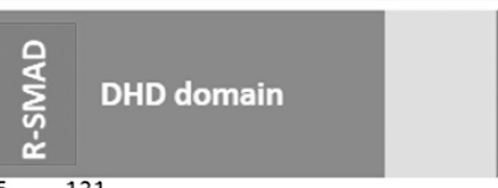

192

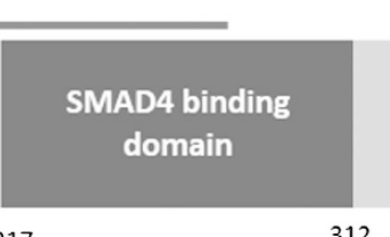

312

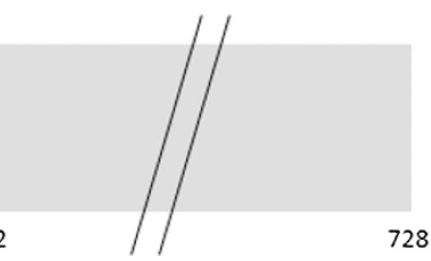

Figure 2 (a) Schematic representation of the SKI domains in exon 1. Location of all SKI mutations known so far with respect to the binding sites of SKIbinding partners. Mutations in red are identified and discussed in this paper. (b) Overview of all published mutations identified so far in SKI. ${ }^{*}$ One patient was identical in Doyle et $a^{10}$ (patient 4) and Carmignac et al ${ }^{11}$ (patient 12), resulting in 33 independent SGS patients with a mutation in SKI. A full color version of this figure is available at the European Journal of Human Genetics journal online. 


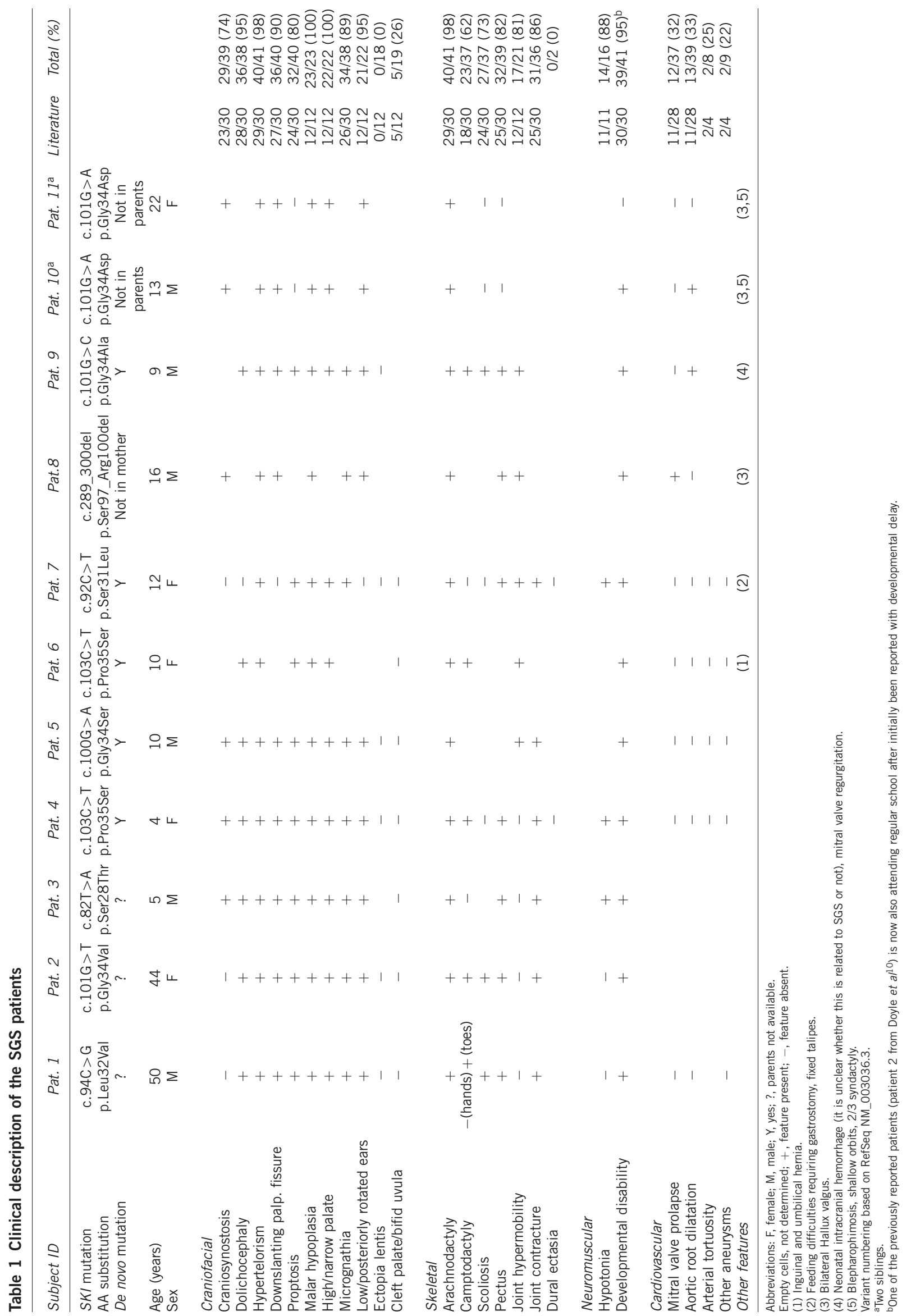


patients in addition to the relatively young age of the patients in the current series. Nevertheless, it is known that the aneurysmal phenotype is usually less severe and less penetrant in SGS cases compared with MFS and LDS cases. ${ }^{2,10}$ Still, lifelong follow-up seems to be warranted. If baseline echocardiography is normal, then we recommend yearly follow-up in children and subsequently every 2-3 years in adults. Besides the low frequency of cardiovascular features, the patients in the current study display the typical craniofacial, skeletal, and neuromuscular features of SGS, including craniosynostosis, arachnodactyly, and developmental and intellectual disability. Importantly, not all patients have intellectual disability as patient 11 in our series is pursuing university level education and one of the previously reported patients (patient 2 from Doyle et $a l^{10}$ ) is now also attending regular school after initially been reported with some developmental delay. As such, the absence of intellectual disability does not preclude the diagnosis of SGS. In addition, severe intellectual disability seems to make a diagnosis of SGS unlikely, as we observed most often mild/moderate intellectual disability.

Dysregulated TGF $\beta$ signaling has clearly been implicated in the pathogenesis of thoracic aortic aneurysm phenotypes. Since SGS shows a major clinical overlap with both MFS and LDS, it was not unexpected to find impaired TGF $\beta$ signaling also at the core of the SGS pathogenesis. Indeed, both canonical and non-canonical TGF $\beta$ signaling were increased in primary dermal fibroblasts from SGS patients, findings that were similar to those observed in MFS and LDS patients. ${ }^{10}$ SKI is a negative regulator of TGF $\beta$ signaling, but has no catalytic activities nor can it bind DNA directly. SKI is dependent on interactions with other cellular partners, such as SMAD proteins, and transcriptional co-regulators including $\mathrm{N}-\mathrm{CoR}, \mathrm{mSin} 3 \mathrm{~A}$, and histone deacetylases. ${ }^{14,16}$ Remarkably, all SKI mutations described so far cluster into two domains: the R-SMAD binding domain, involved in TGF $\beta$ signaling, and the DHD domain, which mediates binding to SNW1 and N-Cor proteins. The latter are essential for the transforming activity of SKI and for the recruitment of transcriptional corepressors. On the one hand, the SKI mutations most likely destroy the binding capacities of these specific domains, leading to the typical MFS- and LDS-like manifestations observed in SGS patients. On the other hand, SKI also has a prominent role in neurogenesis, which may explain why SGS patients show intellectual disability.

So far 17 different SKI mutations have been identified in 33 unrelated patients (1 patient was identical in Doyle et al ${ }^{10}$ and Carmignac et $\left.a l^{11}\right)$. Adding our new findings to the existing data clearly reveals a mutational hotspot, with all mutations localized in exon 1 and with $73 \%$ (24 out of 33 ) of the hitherto described independent patients having mutations affecting a stretch of five SKI residues (from p.(Ser31) to p.(Pro35)). This fact has important implications for molecular testing, which most probably can be restricted to mutation analysis of exon 1 of SKI, or, as this exon is huge, to the first half of exon 1.

In conclusion, we have extended the mutational spectrum of SKI and have revealed a mutational hotspot in SKI, with $73 \%$ of the hitherto described SGS patients having mutations affecting four adjacent residues in the R-SMAD binding domain of SKI: p.(Ser31) p.(Leu32), p.(Gly34), and p.(Pro35).

\section{CONFLICT OF INTEREST}

The authors declare no conflict of interest.

\section{ACKNOWLEDGEMENTS}

DS is supported by a $\mathrm{PhD}$ grant from the Agency for Innovation by Science and Technology. BLL is a senior clinical investigator of the Fund for Scientific Research, Flanders (FWO, Belgium). This research was supported by funding from the Fund for Scientific Research, Flanders (FWO, Belgium) [G.0458.09; G.0221.12], the Fondation Leducq, the European Research Council (ERC), the University of Antwerp (Lanceringsproject), the National Institute for Health Research (NIHR) Biomedical Research Centre based at Guy's and St Thomas NHS Foundation Trust and King's College London.

1 Shprintzen RJ, Goldberg RB: A recurrent pattern syndrome of craniosynostosis associated with arachnodactyly and abdominal hernias. J Craniofac Genet Dev Biol 1982; 2: 65-74.

2 Robinson PN, Neumann LM, Demuth S et al: Shprintzen-Goldberg syndrome: fourteen new patients and a clinical analysis. Am J Med Genet A 2005; 135: 251-262.

3 Renard M, Holm T, Veith R et al: Altered TGFbeta signaling and cardiovascular manifestations in patients with autosomal recessive cutis laxa type I caused by fibulin4 deficiency. Eur J Hum Genet 2010; 18: 895-901.

4 Coucke PJ, Willaert A, Wessels MW et al: Mutations in the facilitative glucose transporter GLUT10 alter angiogenesis and cause arterial tortuosity syndrome. Nat Genet 2006; 38: 452-457.

5 Neptune ER, Frischmeyer PA, Arking DE et al: Dysregulation of TGF-beta activation contributes to pathogenesis in Marfan syndrome. Nat Genet 2003; 33: 407-411.

$6 \mathrm{Ng} \mathrm{CM}$, Cheng A, Myers LA et al: TGF-beta-dependent pathogenesis of mitral valve prolapse in a mouse model of Marfan syndrome. J Clin Invest 2004; 114: 1586-1592.

7 van de Laar IMBH, Oldenburg RA, Pals G et al: Mutations in SMAD3 cause a syndromic form of aortic aneurysms and dissections with early-onset osteoarthritis. Nat Genet 2011; 43: 121-U165.

8 Habashi JP, Judge DP, Holm TM et al: Losartan, an AT1 antagonist, prevents aortic aneurysm in a mouse model of Marfan syndrome. Science 2006; 312 $117-121$.

9 Holm TM, Habashi JP, Doyle JJ et al: Noncanonical TGFbeta signaling contributes to aortic aneurysm progression in Marfan syndrome mice. Science 2011; 332: 358-361.

10 Doyle AJ, Doyle JJ, Bessling SL et al: Mutations in the TGF-beta repressor SKI cause Shprintzen-Goldberg syndrome with aortic aneurysm. Nat Genet 2012; 44: 1249-1254.

11 Carmignac V, Thevenon J, Ades $\mathrm{L}$ et al: In-frame mutations in exon 1 of SKI cause dominant Shprintzen-Goldberg syndrome. Am J Hum Genet 2012; 91: 950-957.

12 Au PY, Racher HE, Graham Jr JM et al: De novo exon 1 missense mutations of SKI and Shprintzen-Goldberg syndrome: Two new cases and a clinical review. Am J Med Genet A 2013; 164: 676-684.

13 Akiyoshi S, Inoue $\mathrm{H}$, Hanai J et al: c-Ski acts as a transcriptional co-repressor in transforming growth factor-beta signaling through interaction with smads. J Biol Chem 1999; 274: 35269-35277.

14 Luo K, Stroschein SL, Wang W et al: The Ski oncoprotein interacts with the Smad proteins to repress TGFbeta signaling. Genes Dev 1999; 13: 2196-2206.

$15 \mathrm{Xu} \mathrm{W,} \mathrm{Angelis} \mathrm{K,} \mathrm{Danielpour} \mathrm{D} \mathrm{et} \mathrm{al:} \mathrm{Ski} \mathrm{acts} \mathrm{as} \mathrm{a} \mathrm{co-repressor} \mathrm{with} \mathrm{Smad2} \mathrm{and} \mathrm{Smad3}$ to regulate the response to type beta transforming growth factor. Proc Natl Acad Sci USA 2000; 97: 5924-5929.

16 Nomura T, Khan MM, Kaul SC et al: Ski is a component of the histone deacetylase complex required for transcriptional repression by Mad and thyroid hormone receptor. Genes Dev 1999; 13: 412-423.

17 Deheuninck J, Luo K: Ski and SnoN, potent negative regulators of TGF-beta signaling. Cell Res 2009; 19: 47-57.

18 Kaufman CD, Martinez-Rodriguez G, Hackett Jr PB: Ectopic expression of c-ski disrupts gastrulation and neural patterning in zebrafish. Mech Dev 2000; 95: $147-162$.

19 Berk M, Desai SY, Heyman HC, Colmenares C: Mice lacking the ski proto-oncogene have defects in neurulation, craniofacial, patterning, and skeletal muscle development. Genes Dev 1997; 11: 2029-2039.

20 Amaravadi LS, Neff AW, Sleeman JP, Smith RC: Autonomous neural axis formation by ectopic expression of the protooncogene c-ski. Dev Biol 1997; 192: 392-404.

Supplementary Information accompanies this paper on European Journal of Human Genetics website (http://www.nature.com/ejhg) 